Service social

\title{
Familles et services sociaux : quelles limites aux interventions?
}

\section{Renée B. Dandurand}

Volume 48, numéro 1, 2001

URI : https://id.erudit.org/iderudit/006874ar

DOI : https://doi.org/10.7202/006874ar

Aller au sommaire du numéro

Éditeur(s)

École de service social de l'Université Laval

ISSN

0037-2633 (imprimé)

1708-1734 (numérique)

Découvrir la revue

Citer cet article

Dandurand, R. B. (2001). Familles et services sociaux : quelles limites aux interventions? Service social, 48(1), 1-15. https://doi.org/10.7202/006874ar

\section{Résumé de l'article}

À l'aide d'une recherche menée auprès de jeunes familles de trois quartiers montréalais différenciés selon le statut socio-économique, l'article présente le point de vue des parents sur les services sociaux. Certaines caractéristiques s'en dégagent : quand il n'est pas ignoré, le professionnel du travail social est représenté comme une figure ou bien d'assistance ou bien de surveillance. Sont ensuite définis quatre espaces d'intervention qui délimitent les marges de manoeuvre entre lesquelles agissent actuellement les travailleurs sociaux. Deux notions centrales guident l'analyse : l'autonomie des familles, de même que la solidarité, privée et publique, qui les entoure. 


\section{Familles et services sociaux : quelles limites aux interventions ${ }^{1}$ ?}

Renée B.-DANDURAND

À l'aide d'une recherche menée auprès de jeunes familles de trois quartiers montréalais différenciés selon le statut socio-économique, l'article présente le point de vue des parents sur les services sociaux. Certaines caractéristiques s'en dégagent : quand il n'est pas ignoré, le professionnel du travail social est représenté comme une figure ou bien d'assistance ou bien de surveillance. Sont ensuite définis quatre espaces d'intervention qui délimitent les marges de manœuvre entre lesquelles agissent actuellement les travailleurs sociaux. Deux notions centrales guident l'analyse : l'autonomie des familles, de même que la solidarité, privée et publique, qui les entoure.

On the basis of research undertaken with young families in three separate socio-economic Montréal districts, a parents view of social services is presented. The following characteristics emerge: when not entirely ignored, the social service professional is seen as either a helpful or a controlling figure. Four levels of intervention are detailed which circumscribe the room for action by social workers. The analysis is guided by two major principles: the autonomy of families and the private and public solidarity supporting them.

1 Le sujet de cet article a d'abord donné lieu à une conférence au congrès annuel de l'ANPASE (Association nationale des professionnels et acteurs de l'action sociale et sanitaire en faveur de l'enfance et de la famille), à Aix-enProvence, en France, du 20 au 23 octobre 1998. C'est la raison pour laquelle ce texte comporte certaines références à la France. 


\section{LA FAMILLE IDÉALE : UNE FAMILLE AUTONOME}

De nos jours, quand on parle de famille « idéale », le débat sur le sujet consiste généralement en une discussion sur les mérites respectifs de la famille selon son statut légal ou sa composition: doit-elle être légitime ou peut-elle être naturelle? Doit-elle être biparentale « intacte », ou peut-elle être monoparentale ou recomposée? J'aimerais répondre autrement à cette question et poser que dans les sociétés occidentales du $X X^{\mathrm{e}}$ siècle la famille idéale est une famille nucléaire qui se veut autonome par rapport à son entourage, donc qui s'oriente et agit par elle-même.

C'est dans la bourgeoisie montante du XVII ${ }^{e}$ siècle que les historiens placent les premières manifestations d'une famille moderne (Shorter, 1977). Cette famille, qui est restreinte et prétend se situer en marge de la famille étendue et de la communauté ambiante, se replie sur un espace privé, celui de la maisonnée. C'est là qu'après un choix qui deviendra de plus en plus libre d'unir leur destinée les époux élèvent leurs enfants, en assument le soin, l'éducation et l'entretien selon des normes nouvelles qu'on peut ainsi résumer : l'enfant est de moins en moins vu comme un adulte en miniature, mais comme un être à part entière, un individu dont il importe de développer les potentialités et à qui il faut prodiguer amour et protection (Ariès, 1960). À tendance nucléaire et autonome, cette forme familiale, présente dans la bourgeoisie, correspond à un idéal nouveau qui sera, jusqu'à aujourd'hui, transmis aux familles des autres milieux sociaux, les classes moyenne et ouvrière.

\section{UNE AUTONOMIE TOUTE RELATIVE : LES SOLIDARITÉS PRIVÉES ET PUBLIQUES}

Mais cette famille autonome est bien un idéal, un idéal tout à fait en continuité avec le désir d'authenticité et de liberté de l'individu moderne (Taylor, 1998). En réalité, dans l'exercice du parentage, c'est-à-dire des fonctions d'entretien, de soin et d'éducation des enfants en vue du renouvellement du patrimoine humain, les familles nucléaires contemporaines sont diversement relayées par deux formes de solidarité : d'abord, par des soutiens privés, en particulier par la famille élargie qui, bien que transformée depuis 
le dernier siècle, tient toujours une place importante dans le paysage relationnel de nos contemporains; ensuite, par des aides publiques mises en place par l'État (dans les domaines de l'éducation, du sociosanitaire, de l'assistance sociale ou fiscale) ou par divers organismes de la société civile (bénévoles, groupes religieux, communautés locales, etc.).

A. Les solidarités publiques sont devenues plus visibles quand les États modernes se sont faits "providence ». Dès le XIX siècle en France (un peu plus tardivement en Amérique du Nord), sont mis en place des services et protections pour les plus vulnérables : enfants et mères seules, personnes âgées, malades, handicapées, accidentées et exclues du travail.

$\mathrm{Si}$, en France, la protection de la famille et des enfants est parmi les plus précoces en Occident, au Canada l'implantation de mesures de solidarité publique pour les familles est plus récente et plus timide. Bien que le suivi sanitaire et la protection des enfants datent du tournant du siècle au Québec, la loi d'obligation scolaire est tardive (1943) et les premières allocations familiales datent de l'après-deuxième-guerre. Si l'on excepte les années de guerre, le développement durable de services de garde s'amorce pendant la décennie 1970 seulement, et c'est en 1987 que notre province se donne une politique familiale explicite (Dandurand et Ouellette, 1995a). En Amérique du Nord, le Québec est le seul territoire, parmi les provinces canadiennes et les États américains, à disposer d'une telle politique.

B. On a pensé, au milieu du $X X^{e}$ siècle, que les solidarités publiques avaient remplacé et même fait disparaître les solidarités privées, en particulier celles qui provenaient de la famille élargie. Les études menées depuis la fin des années 1970, aussi bien en France (Pitrou, 1992; Dechaux, 1994) qu'au Québec (Fortin, 1987; Dandurand et Ouellette, 1992; Godbout et Charbonneau, 1996), montrent que les liens et les échanges avec la parenté immédiate, s'ils se sont transformés, sont toujours bien présents.

Dans la plupart des pays occidentaux, les caractéristiques des solidarités de parenté sont assez similaires. En France, ces 
échanges privés totalisent chaque année plusieurs milliards de francs, dont les trois quarts sont en espèce. En France comme au Québec, les dons proviennent surtout de la génération " pivot ", celle des jeunes grands-parents, et ce sont les femmes qui en sont les principales artisanes. Les soutiens accordés sont en général ponctuels et de courte durée. En milieu aisé, les échanges se font davantage sous forme de biens, en milieu modeste, sous forme de services. Dans nos deux pays, les analystes s'entendent pour soutenir que les échanges familiaux sont fragiles et inégalitaires et ne peuvent, sans être mis en cause, se substituer aux solidarités publiques (voir notamment Théry, 1998, p. 91).

C'est donc dire que, outre les parents, de nombreux acteurs interviennent dans l'entretien, le soin et l'éducation des enfants d'aujourd'hui : l'autonomie des familles à cet égard est ainsi toute relative. Par exemple, les activités de parentage doivent se conjuguer quotidiennement avec l'influence et les exigences de l'école comme avec les critères de santé publique et de protection de l'enfant; ces activités de parentage s'associent également au soutien des grands-parents, par exemple pour la garde ou le soutien financier. Et si la famille élargie n'est pas disponible, les aides publiques de l'État ou du marché devront prendre le relais. Dans les situations critiques, quand les familles n'ont pas ou n'ont plus les ressources personnelles ou financières pour soutenir cette autonomie relative, l'intervention sociale se fait de plus en plus présente. C'est alors qu'entrent en scène, notamment, les spécialistes du travail social.

\section{LE TRAVAIL SOCIAL À L'INTERSECTION DES SOLIDARITÉS AUPRÈS DES FAMILLES}

Dans l'exercice de l'action publique auprès des familles, les travailleurs sociaux ${ }^{2}$ sont les professionnels qui occupent une position parmi les plus stratégiques car, justement, elle se situe à l'intersection des solidarités publiques et privées. À cet égard, on peut en effet concevoir qu'après avoir cerné et pris en compte les difficultés des familles rencontrées, l'essentiel de leur travail

2 Le générique masculin est utilisé sans discrimination et pour alléger le texte. 
consiste en l'exercice de deux fonctions principales. Dans un premier temps, il s'agit d'évaluer les ressources privées des familles et de se demander si, avec l'aide de leurs proches, les parents ont la capacité d'exercer, selon les normes reçues, leurs responsabilités à l'égard de leurs enfants. Dans un deuxième temps, les travailleurs sociaux se demandent s'il y a lieu de compléter l'action des familles ou s'il faut aller jusqu'à s'y substituer; ils exercent alors une fonction de relais vers des ressources externes à la famille, en particulier celles de l'État, qui constituent l'essentiel des solidarités publiques.

Pour en arriver à saisir s'il y a des limites aux interventions des travailleurs sociaux auprès des familles, je vais présenter certains résultats d'une recherche qualitative ${ }^{3}$ menée à Montréal, au début des années 1990, auprès de jeunes familles (les parents ont en moyenne 35 ans) de trois quartiers, différenciés selon l'appartenance socio-économique.

\section{UNE ENQUÊTE MONTRÉALAISE AUPRÈS DES JEUNES FAMILLES}

Notre étude visait à cerner la situation des familles avec charge d'enfants par rapport à leurs parentèles et aux autres réseaux potentiels de soutien, notamment lors de certains moments clés de la vie familiale: installation du foyer, naissances, retour des mères sur le marché de l'emploi, chômage, maladies, problèmes graves des enfants et des adultes et, enfin, ruptures conjugales. Les données ont été recueillies à l'aide d'entretiens semi-dirigés auprès de 60 foyers montréalais biparentaux et monoparentaux ${ }^{4}$, répartis dans trois quartiers socio-économiquement contrastés: Saint-Henri, où habitent des ouvriers peu qualifiés, des employés

3 Recherche menée en collaboration avec F.-R. Ouellette dans le cadre des travaux du chantier Familles, sexe et générations de l'Institut québécois de recherche sur la culture (voir Dandurand et Ouellette, 1992 et 1995). Cette recherche a reçu une subvention du Conseil québécois de la recherche sociale.

4 Nous avons fait 20 entrevues par quartier, en sélectionnant un nombre de foyers monoparentaux qui correspondait au pourcentage de ces foyers parmi les familles parentales de l'ensemble du quartier, selon les données de recensement disponibles : Saint-Henri, environ $45 \%$, Rosemont, environ $35 \%$ et Outremont, environ $25 \%$. 
des services et des assistés sociaux; Rosemont, où logent des ouvriers qualifiés ainsi que des employés, techniciens et petits commerçants; enfin Outremont, où résident des professionnels et des cadres. Ces 60 foyers avaient tous au moins un enfant inscrit à l'école primaire, c'est-à-dire ayant entre 5 et 11 ans. Ils ont d'ailleurs été recrutés en passant par les écoles de quartier.

J'aimerais insister sur deux aspects des résultats de cette enquête : d'une part, le caractère inégalitaire des soutiens ou solidarités privées, qui fragilise l'autonomie des familles peu favorisées et nécessite souvent l'exercice des solidarités publiques; d'autre part, le point de vue de ces familles sur les interventions des travailleurs sociaux.

\section{A. Le caractère inégalitaire des solidarités privées}

Tous les foyers de notre enquête ne disposaient pas des mêmes ressources pour ce qui est des solidarités privées. On sait que l'entraide dans la famille élargie est surtout le fait de la "génération pivot ", celle des grands-parents appelés à soutenir les générations d'adultes plus jeunes et plus âgés qu'eux. Dans notre enquête, la génération pivot est diversement présente : si les trois quarts des grands-parents du quartier aisé sont encore vivants, c'est le cas des deux tiers en milieu intermédiaire et de la moitié seulement en milieu populaire. Cette première inégalité, qui rejoint les statistiques de l'espérance de vie, s'accentue quand la résidence des grands-parents donateurs n'est pas située à proximité. En effet, la distance entre les apparentés importe beaucoup moins en milieu aisé qu'ailleurs, d'abord parce que la nature des échanges (des biens plutôt que des services) n'exige pas la proximité des résidences; ensuite parce que le coût des communications et des rencontres (appels interurbains, déplacements) est abordable pour eux. II en est tout autrement en milieu modeste, là où les échanges se font davantage sous forme de services, ce qui exige donc la proximité des résidences; là également où les communications et rencontres, coûteuses quand on vit à distance les uns des autres, sont inévitablement beaucoup plus rares. Et même quand la parenté est disponible, son aide est forcément limitée à ses propres ressources : un coup de main, un hébergement temporaire, la garde passagère des enfants, mais certes pas les secours à long terme en cas de chômage ou de rupture 
conjugale. Le soutien en provenance des solidarités publiques doit donc rapidement prendre le relais ${ }^{5}$.

Les inégalités sont encore présentes quand on examine l'ensemble du réseau de sociabilité des familles de notre enquête, soit non seulement les liens avec la parenté, mais aussi avec les amis, les voisins et les collègues de travail. À cet égard, les trois quartiers de notre enquête présentaient des modèles de réseaux relationnels différents.

En milieu populaire, deux modèles de réseaux ont été observés : dans les familles biparentales actives sur le marché du travail, le réseau des personnes interrogées est presque uniquement composé de liens de parenté, auxquels s'ajoutent quelques liens d'amitié chez les femmes; en général, ni les voisins ni les collègues de travail ne pénètrent dans l'intimité de la maisonnée. Dans le même quartier, les autres familles (la plupart monoparentales et privées de parenté résidant à proximité) vivent de prestations d'assurance-emploi ou d'assistance sociale; elles présentent un autre modèle de réseau fait de liens personnels et familiaux épars, parfois puisés à même la vie du voisinage et des groupes communautaires locaux (Dandurand et Ouellette, 1995b).

En milieu intermédiaire et aisé, les liens avec les voisins, les collègues de travail et les amis prennent de plus en plus de place. Pour ces familles avec jeunes enfants, la parenté conserve une certaine importance, mais elle ne prend pas toute la place. Le quartier le plus fortuné présente les réseaux les plus diversifiés, offrant des liens nombreux et étendus dans l'espace social, ce qui permet à ces familles d'établir une connexion souvent directe avec des ressources aussi essentielles, par exemple, que les soins de santé : un voisin est pédiatre, le beau-père est médecin ou cadre dans un centre hospitalier, le psychologue est un collègue de travail d'un ami. L'orientation vers les services sociosanitaires et scolaires, indispensables à la vie des jeunes familles, se fait par des voies familières et privées, ce qui n'est pas du tout le

5 Dans les temps de crise du travail, les familles de milieu intermédiaire ou même favorisé peuvent aussi se retrouver dans une situation critique. Mais si elles sont dotées d'un réseau relationnel et familial plus aisé, elles pourront éviter de basculer dans la pauvreté. 
cas des familles de milieu populaire qui ne disposent pas de telles recommandations. De plus, si les services publics ne sont pas satisfaisants ou immédiatement accessibles, ces familles peuvent facilement recourir aux ressources privées du marché qui, en Amérique du Nord, sont une solution de rechange toujours offerte à ceux qui en ont les moyens.

La comparaison des jeunes familles de différents milieux sociaux permet de voir à quel point les solidarités privées ont un caractère inégalitaire, à quel point aussi est fragile l'autonomie des foyers de milieu modeste. Ce sont là des éléments essentiels à la compréhension de la culture des familles et, en particulier, des représentations que les parents se font de l'intervention des services sociaux.

\section{B. Le point de vue des familles sur les interventions des travailleurs sociaux}

Trois figures de travailleurs sociaux ont été observées dans les représentations que nos familles véhiculent.

a) Dans les familles les plus favorisées de notre enquête, l'intervenant des services sociaux est une figure presque ignorée. Tout au plus accepte-t-on de le consulter pour les problèmes des adolescents (fugue, drogue) et, bien sûr, pour le placement des handicapés et des personnes âgées. Pour les autres problèmes de la vie, notamment autour des dépressions ou des ruptures conjugales, on s'adresse directement aux thérapeutes du psychique qu'on juge plus spécialisés, donc mieux armés pour venir en aide, mais dont les services ne sont généralement pas gratuits. Par ailleurs, ces milieux scolarisés connaissent mieux les ressources collectives, savent faire valoir leurs droits et se débrouillent assez bien dans le dédale bureaucratique des services sociosanitaires de l'État (Pitrou, 1992). Enfin, on l'a vu, les réseaux personnels de ces familles comprennent souvent des parents, amis, collègues ou voisins qui occupent des postes de travail à proximité de tels services.

En somme, dans les familles de milieu aisé, l'intervenant des services sociaux est peu présent. Sa fonction de conseil et de 
relais vers les services des établissements publics est plus rarement utilisée qu'en d'autres milieux du fait des ressources que tirent les familles de leur réseau personnel, de leur capacité à mener elles-mêmes leurs démarches et à recourir aux ressources privées, généralement payantes.

b) II en est tout autrement dans les familles de milieu populaire et même dans celles de milieu intermédiaire quand ces dernières doivent faire face à des problèmes graves (perte d'emploi, alcoolisme, drogue ou tentative de suicide). Parce qu'elle est moins spécialisée et plus polyvalente, moins parcellaire et plus globale, l'intervention du travailleur social est très valorisée. Le mode de résolution des problèmes, qui est le propre de cette intervention et qui fait appel aux solutions concrètes et au groupe familial plutôt qu'aux ressources personnelles des individus, est explicitement préféré à l'approche du psychothérapeute, moins accessible en service public. En outre, on estime que le psychologue dérange, car " il vient nous chercher en dedans "; quant au psychiatre, il prescrit des calmants ou des antidépresseurs qu'on juge nocifs et qu'on dit refuser de prendre.

Quand le travailleur social est considéré de façon positive, il est donc vu comme une véritable figure d'assistance avec laquelle se noue une collaboration, que l'intervention soit de courte ou de longue durée : cela va de l'inscription des enfants en colonie de vacances jusqu'à un counseling hebdomadaire qui, dans certains cas, peut s'étendre sur un bon nombre d'années. Quand il est requis, le placement temporaire des enfants en famille d'accueil est considéré comme une façon de "soulager la mère " plutôt que de lui "enlever ses enfants ". La relation entre la travailleuse sociale (la plupart du temps, c'est une femme) et la mère (souvent en foyer monoparental) est parfois jugée si nécessaire que la famille évite de déménager dans un autre quartier de peur de perdre sa travailleuse sociale. On mentionne des relations presque intimes avec celle-ci : " je l'appelle Suzanne », "c'est une amie », c'est « comme une sœur ». À plusieurs reprises, les travailleurs sociaux incitent les familles, surtout celles des mères seules, à s'inscrire aux activités des organismes de loisir pour jeunes, des garderies publiques, des comptoirs alimentaires et des 
coopératives de vêtements ou, encore, des centres de rencontre ou de formation. Dans ces milieux, la plupart des intervenants des services sociaux tentent de travailler en lien étroit avec la communauté.

c) C'est parmi quelques familles ouvrières mais surtout dans les familles monoparentales assistées des secteurs les plus défavorisés qu'on peut observer une vision négative de l'intervenant en travail social : celui-ci est vu comme une figure de surveillance, qui menace la vie des familles ${ }^{6}$. La hantise de "se faire enlever ses enfants" par une travailleuse sociale est toute présente dans la vie de ces mères seules. Selon elles, cette méfiance est inscrite dans leur propre histoire, personnelle et familiale. Ainsi, plusieurs d'entre elles ont été, enfants, soumises à un placement en famille d'accueil. Plus tard, enceintes alors qu'elles étaient adolescentes ou mères élevant seules trois ou quatre enfants, elles auraient été incitées par une travailleuse sociale à interrompre leur grossesse. Ou encore, pauvres et isolées, elles auraient reçu un «bon conseil » d'une voisine: "Tiens-toi loin des travailleuses sociales si tu veux pas avoir de troubles ${ }^{7}$. "

Voilà donc des familles qui, autant que possible, évitent de faire appel aux services sociaux. Parce qu'elles craignent d'être " dénoncées ", elles fréquentent le moins possible les services de garde à l'enfance et se présentent avec méfiance aux services sanitaires. La suspicion s'étend même aux centres bénévoles de quartier destinés aux familles. II semble qu'une seule ressource échappe à ce qui est ainsi perçu comme un cercle de contrôle des services sociaux: quelques organismes mis en place par des congrégations religieuses, qui assurent des services de garde de la petite enfance et de surveillance des devoirs après la classe.

Une image dramatique des services sociaux vus comme figure de surveillance nous a été donnée au Canada en mars 1998. Une

6 Moins présente que le précédent modèle dans notre enquête, cette représentation a été largement observée dans une étude sur des mères de milieu défavorisé de Montréal (voir Colin et collab., 1992).

7 Avoir des ennuis. 
mère amérindienne de la province de l'Alberta, Connie Jacobs, s'est opposée violemment aux services qui voulaient placer ses enfants en milieu d'accueil : à l'aide d'une carabine, elle a visé le travailleur social et le policier qui se présentaient à sa résidence. Le policier a riposté et l'a tuée ainsi qu'un de ses enfants. Les clientèles amérindiennes sont certes des populations très marginales dans notre pays. Mais de plus en plus de pays ont des populations "à la marge » : on pense, pour les États-Unis, aux populations noires, chicanos et amérindiennes, pour la France, aux réfugiés d'Afrique et d'Europe de l'Est. Ce que dénote l'affaire Jacobs, ce n'est pas seulement une attitude d'évitement des services sociaux, comme celle qui a été décrite précédemment, mais une attitude tout à fait hostile et agressive.

\section{QUELLES LIMITES AUX INTERVENTIONS AUPRÈS DES FAMILLES?}

On en revient à la question centrale de cet article : quelles sont les limites des interventions des travailleurs sociaux auprès des familles? En d'autres termes, quelles sont leurs marges de manœuvre, entre quelles bornes se situent leurs espaces d'intervention? Quels enjeux sont ainsi soulevés et quels dilemmes sont vécus par ces professionnels? J'y répondrai en quatre points qui délimitent ce qu'on pourrait appeler des espaces d'intervention.

\section{$1^{\text {er }}$ espace d'intervention :}

\section{Entre l'érosion de la protection sociale de l'État et l'augmentation des besoins des familles}

Dans la plupart des pays occidentaux, l'État redéfinit et restreint son rôle protecteur, alors que sous l'effet de la précarisation du travail et des transformations du lien familial les besoins de soutien des familles sont de plus en plus grands. C'est là un contexte qui non seulement augmente la fragilité et l'inégalité des familles, mais qui contribue à rétrécir la marge de manœuvre des travailleurs sociaux auprès d'elles. Par exemple, si ces intervenants voient diminuer le temps à accorder à chaque dossier, la qualité de la relation avec la clientèle, nécessaire (on l'a vu précédemment) à une intervention efficace, risque d'être compromise. 


\section{$2^{\mathrm{e}}$ espace d'intervention :}

Entre le recul des solidarités publiques et l'importance de ne pas épuiser ni tarir les ressources des solidarités familiales

Si la fragilité des solidarités familiales et les inégalités qu'elles recèlent s'ajoutent au contexte de crise du travail et de la famille qui touche les pays occidentaux, on doit admettre que le filet de sécurité de l'État doit être tenu comme tout à fait indispensable. Dans son rapport à la ministre de la Justice ainsi qu'à la ministre de l'Emploi et de la Solidarité, la sociologue française Irène Théry (1998, p. 91) se prononce sans équivoque sur le sujet: "Toute interprétation des solidarités privées comme substituts possibles au recul des solidarités publiques mettrait en cause le système global des échanges entre générations. ॥

II importe donc de prévoir les effets à long terme de cette dynamique. Non seulement l'épuisement des solidarités familiales aurait pour conséquence des coûts supplémentaires pour l'État (en soutien ou en contrôle des populations), mais cela représenterait surtout l'altération sinon la destruction du lien familial, un lien social fondamental pour la sécurité des êtres humains. Les travailleurs sociaux sont les professionnels parmi les mieux placés pour préserver les solidarités familiales et faire en sorte que l'effort demandé aux familles dans le soutien de leurs proches ne dépasse pas les ressources personnelles et matérielles des donateurs. Ils ont donc un rôle tout à fait stratégique dans le maintien d'un équilibre entre les solidarités publiques et privées.

\section{$3^{\mathrm{e}}$ espace d'intervention :}

Entre le respect de l'autonomie des familles et la responsabilité de protection des plus vulnérables

II me semble que cette marge de manœuvre des travailleurs sociaux représente l'espace de responsabilité le plus lourd sur le plan éthique: tout en respectant l'attitude des familles à leur égard, qu'elle soit empreinte d'indifférence, de collaboration, de méfiance, voire d'hostilité, les travailleurs sociaux doivent garantir la protection des membres les plus vulnérables des familles. Cet article a insisté sur le point de vue des familles et tenté de faire 
comprendre sur quelles logiques socio-économiques et culturelles s'appuient les attitudes des familles à l'égard des intervenants du travail social. Je n'ai cependant pas voulu sous-estimer l'importance de la responsabilité des intervenants dans la nécessaire protection des plus fragiles.

\section{$4^{\mathrm{e}}$ espace d'intervention :}

\section{Faut-il aller vers l'éducation familiale, en particulier vers l'éducation des parents dits démissionnaires ${ }^{8}$ ?}

Devant la diminution des fonds sociaux de l'État pour résoudre les problèmes accrus des familles, devant la nécessité de ne pas " épuiser " les solidarités familiales, une éducation formelle des parents est une solution de plus en plus proposée, autant par les services scolaires et sociosanitaires que par les associations familiales : ne faut-il pas aider les parents à mieux assumer leurs responsabilités, les aider à développer leur "compétence » parentale (un terme cher aux experts) ou leurs " habiletés » parentales (un terme que préfèrent les associations familiales)?

Éduquer les familles afin que leurs fonctions parentales soient mieux exercées, afin que par conséquent l'autonomie des familles soit mieux affirmée, qu'elles s'en sortent mieux tout en dépendant moins des solidarités publiques, ce sont là des objectifs louables et certes souhaitables. Sont-ils réalisables? Et dans quelles conditions? Je n'ai pas d'idée très arrêtée sur le sujet, mais j'aimerais formuler quelques questions.

Dans certains cas de négligence ou d'agression parentale graves, l'éducation suppose un changement fondamental du mode de relation parents-enfants : est-ce là un apprentissage possible? Le contenu de l'éducation proposée correspondra-t-il aux traditions et à la culture familiales? Est-il envisageable d'arriver à éduquer sans introduire auprès des familles un suivi sinon une surveillance

8 L'expression « démissionnaire » est souvent utilisée en France pour qualifier des parents des banlieues marquées par la délinquance des adolescents et des jeunes. L'expression est reprise au Québec où des articles récents parlent de la "démission des familles". Voir Michel Venne, "Raccrocher les familles », Le Devoir, 19 janvier 1999, p. A-6. 
qui risque d'augmenter, là où elle s'observe, la méfiance à l'endroit des intervenants? Les pouvoirs administratifs et législatifs ne seront-ils pas tentés d'aller au-delà d'une "éducation » et d'exercer une intervention de type "sanction " face aux parents jugés "démissionnaires »? (C'est ce que les États-Unis ont fait récemment en retirant l'assistance sociale aux parents d'adolescents « délinquants » qui ne fréquentaient plus l'école.)

Une solution comme l'éducation parentale, et surtout celle des parents dits démissionnaires, qui apparaît aujourd'hui comme une panacée, peut dans certains cas avoir pour effet d'augmenter l'autonomie souhaitée des familles, mais elle peut aussi avoir des effets négatifs sur lesquels il importe de réfléchir maintenant.

Janvier 1999

Renée B. DanduRAND, $P h . D$. Professeure-chercheure INRS-Urbanisation, Culture et Société

\section{Références bibliographiques}

ARIĖS, P. (1960). L'enfant et la vie familiale sous l'ancien régime, Paris, Plon.

Collin, C., F. Ouellet, G. Boyer, C. MARTIN (1992). Extrême pauvreté, maternité et santé, Montréal, Saint-Martin.

DANDURAND, R. B. et F.-R. OuelletTe (1995a). « Famille, État et structuration d'un champ familial », Sociologie et sociétés, vol. XXVII, $n^{\circ} 2$, p. 103-119.

DANDURAND, R. B. et F.-R. Ouellette (1995b). « Le quartier comme espace de vie des familles en milieu populaire ", dans B. Bawin-Le Gros, R. B.-Dandurand, J. Kellerhals et F. de Singly (dir.), Les espaces de la famille, Liège, Médiatique, p. 173-183. 
DANDuRAnd, R. B. et F.-R. Ouellette (1992). Autonomie et solidarité. Parenté et soutien dans la vie de jeunes familles montréalaises. Rapport de recherche déposé au Conseil québécois de la recherche sociale, Montréal, Institut québécois de recherche sur la culture, $432 \mathrm{p}$.

DechauX, J.-H. (1994). "Les échanges dans la parenté accentuent-ils les inégalités? », Sociétés contemporaines, $\mathrm{n}^{\circ} 17$.

ForTin, A. (1987). Histoires de familles et de réseaux, Montréal, SaintMartin.

Godbout, J. et J. Charbonneau (1996). La circulation du don dans la parenté, Montréal, INRS-Urbanisation.

PITROu, A. (1992). Les solidarités familiales, Toulouse, Privat.

SHORTER, E. (1977). Naissance de la famille moderne, Paris, Seuil.

TAYLOR, C. (1998). Les sources du moi. La formation de l'identité moderne, Montréal, Boréal.

THÉRY, I. (1998). Couple, filiation et parenté aujourd'hui. Le droit face aux mutations de la famille et de la vie privée. Rapport à la ministre de l'Emploi et de la Solidarité et au garde des Sceaux, ministre de la Justice, Paris, La Documentation française et Odile Jacob.

\section{Notes biographiques}

Renée B.-Dandurand est diplômée en philosophie et en anthropologie. Chercheure depuis une vingtaine d'années à l'Institut québécois de recherche sur la culture, puis à l'Institut national de la recherche scientifique, elle s'est intéressée à divers aspects des transformations de la vie familiale, notamment au mariage, à la monoparentalité féminine, aux liens de parenté et aux rapports entre les familles et les institutions étatiques des domaines scolaire et sociosanitaire. Elle est, depuis 1995, directrice scientifique du partenariat de recherche Familles en mouvance et dynamiques intergénérationnelles. 